\title{
Improve the immersion of equipped with virtual maintenance training system to more close to the equipment maintenance training environment
}

\author{
Fushuan $\mathrm{Wu}^{1, \text { a }}$, Jianren $\mathrm{Wang}^{1}$, Yuzhuo Zhao ${ }^{2}$ and Shiliang $\mathrm{Wu}^{3}$ \\ ${ }^{1}$ Wuhan institute of machinery, Wuhan, 430075, China \\ ${ }^{2}$ Wuhan University, Wuhan, 430075, China \\ ${ }^{3}$ China southern Airlines, Guangzhou, 510800, China. \\ a1286697@qq.com
}

Keywords:virtual, reality, technology; equipment; maintenance; immersion

\begin{abstract}
There is the lack of immersion in the equipped with virtual maintenance training system based on the virtual reality technology, which leads to the learners can't understand and grasp the learning content accurately and comprehensively, influencing the effect of teaching and training. In this paper, from the visual immersion, hearing immersion, touch immersion, direction immersion, expression immersion, language immersion six aspects to discuss how to improve the immersion of virtual maintenance training system.
\end{abstract}

\section{Introduction}

With the rapid development of advanced simulation technology such as virtual reality, in the army's teaching and training, equipped with virtual maintenance training system based on the virtual reality technology is going to be "generation with soft and hard, with virtual to real mixing" of a new mode of teaching and training, it is widely used in the training and teaching, playing an important role in improving the teaching and training quality.

However, some existing equipment virtual maintenance training systems, there are still problems with the lack of immersion,a lot of real equipment maintenance information cannot be fully presented,leading to the learners can't understand and grasp the learning content accurately and comprehensively,impact the effect of teaching and training.Enhancing the immersion design of equipment virtual training system, can promote the students' flow experience, making it become closer to equipment maintenance training environment, play the subjective initiative of students' cognition, better close to the practical requirements.

The so-called immersion refers to a realistic felling that allows users to generate their own seem to have exposure to the virtual environment, and can perceive and manipulate various objects in the virtual world, but also to actively participate in various events.

Practice shows that immersion produced mainly from the human perception system and the behavior system. Perception system consists of human vision, hearing, touch, smell and taste and so on; behavior system consists of human posture, orientation, motion, movement, expression and language. In equipment virtual maintenance training, not all of human perception system and the behavior system can be simulated. Mainly in the visual, auditory, tactile three aspects of perceptual system , and direction, expression, language three aspects of behavior system in the of successful virtual.

Therefore, upgrade immersion of equipment virtual maintenance training system, mainly from the visual immersion, hearing immersion, tactile immersion of perception system and direction immersion, expression immersion,language immersion of behavior system three aspects of thinking. 


\section{Method of enhancing the perception system immersion}

\section{To enhance the visual immersion}

Equipment Virtual Maintenance Training System development is to establish realistic model by collecting a variety of data of real equipment, and use three-dimensional graphics construct virtual scene. When the virtual scene construction, enhance the visual immersion should focus on the model establishment of a scene, the sense of space design, light-efficient design and texture design.

1)Set up model(modeling)

Modeling is the key to produce virtual maintenance training system. It mainly includes geometry-based modeling and image-based modeling.

Geometry modeling usually use 3dmax, Maya and other three-dimensional modeling software. Models include high-precision models and low accuracy of the model. The advantage of high-precision model is richly detailed, edges smooth, but the drawback is its too many faces, large files, and slower running. The disadvantage of low accuracy of the model is its low precision, but it can use mapping to achieve realistic effects, with a small file size, fast running advantage. The main components can take high-precision model modeling, while sub-components by low accuracy modeling method, so that can guarantee the results, at the same time, can reduce the complexity of the scene in real-time play-dyed, optimize the number of model faces, improve running speed . For example, when the self-propelled howitzers virtual maintenance training system scenario modeling, distant objects can be built low accuracy of the model, close objects can be built with high precision models.

Image-based modeling refers to using the real picture to construct a virtual model, it does not depend on the geometric model, but the use of the obtained photographed panorama image generation, its rendering speed are independent of scene complexity, with respect to the geometric modeling, it get rid of the conflict between complexity,realism and speed based on geometric modeling , to facilitate real-time generation of complex, realistic virtual models. It generates a small amount of data, computer configuration requirements is low. But by the image-based three-dimensional model has only a visual sense, without real three-dimensional form, so that there is not strong interaction limitations. When modeling the geometry-based modeling and image-based modeling should be combined to achieve flexibility, to effectively address the contradictions that the Virtual Reality Modeling encountered, between realism, running speed. Experience of the author is to use a simple three-dimensional geometric model constructed to meet the interactivity; use multi-angle photographs of the model texture and bump texture, optical effects, color and other treatment, to get a simple,realistic,exquisite detail,rendering fast,highly interactive and realistic model, so designed to produce a virtual scene with relatively strong sense of immersion. When self-propelled howitzer as virtual maintenance training system scenario modeling, object interaction and movement can use geometric modeling, environmental and non-moving objects can be modeled using a map.

2)Sense of space design

Under the premise of realistic modeling effects, construct a good sense of space, to further enhance the visual immersion of virtual maintenance training system. Sense of space generated mainly from the scene geometry perspective and aerial perspective relationship between objects. To ensure the geometric relationships between objects perspective, in the virtual scene, camera parameter settings should be used to meet the vision and perspective of the human eye when viewing an object, making the objects in the virtual scene in line near bigger the far smaller, near low the far high, near coarse the far fine,near wide the far narrow of the law. In order to ensure air relations perspective, the sense of space design should pay attention to environmental atmospheric rendering selection mode, so that objects in the scene near comply gorgeous, distant dark, near clear and distant fuzzy rule. In addition, the virtual scene, we should pay attention to the use of virtual scene detection technology to prevent the emergence of mutual crossing between the object that does not meet the common sense of the situation, ensuring the authenticity of the sense of space. When constructing a virtual environment such as the battlefield, may be represented by fog weather conditions to improve battlefield environment to simulate real sense of immersion. 


\section{3)Light Efficiency Design}

Light effects can express different environment and atmosphere, light-efficient design is another important factor affecting the virtual maintenance training system visual immersion. 3Dmax, Maya and other software can simulate the effect of different light source light on the object, and its rendering engine can real-time present the object surface's color and brightness,resulting in a true natural effect. Such as when the virtual maintenance training system is produced, by using point light, tube light, surface light source,light shadows,light attenuation and so on, make full use of light effects design to heighten the atmosphere of the scene, in order to create a better fit with the theme of expression needs atmosphere and mood to enhance the visual immersion.

4)Texture design

Good design can fully reflect the realistic texture detail of components, can enhance the visual immersion of virtual maintenance training system effectively. Virtual maintenance training system using geometric model can perform delicate texture of the complex structure of parts, often require up to hundreds of thousands of patches, otherwise it is difficult to adapt to the needs of real-time rendering. In the geometric modeling foundation of basic body parts on the use of mapping, can be given a realistic texture and delicate texture of the object, without increasing the complexity of the model can show more details. When surveying techniques for generating components through panoramic three-dimensional effect, the high-resolution camera on the basis of the photos you can handle light color rendering more realistic texture. For example, self-propelled howitzers virtual maintenance training system of virtual component design, the different materials such as copper, wood, rubber, steel, cloth and so on, should be texture designed according to the properties of different materials.

In addition, in order to enhance visual immersion of equipment virtual maintenance training system, but also should make the right choice for terminal display device. The higher the quality of the equipment display image processing, faster image recognition capabilities, the system is stronger in discriminating images , more complete in understanding, the resulting visual immersion is better as possible. Generally, we should choose stereoscopic displays, which allows the user to obtain an object "depth" information that can not be obtained on a flat panel display, and can be observed through the eyes of different images to produce observed parallax, resulting in three-dimensional simulation of visual effects.

\section{Auditory immersion upgrade}

In equipment virtual maintenance training system, auditory immersion mainly through the virtual sound information. Virtual sound information from the form can be divided into voice messages and non-voice information. Voice information refers to human voices. Non-voice information refers to all the other audio information in addition to human language. Voice messages to enhance auditory immersion mainly through tone, intonation, even stop, stress, rhythm softly and organic changes.Non-voice information to create a different state of mind and mood to improve auditory immersion through melody, charm and strength. Ethereal voice can create a sense of technology, echo can create a sense of open space, deep voice can create a mysterious atmosphere, cheerful music gives a sense of movement, soothing sounds can create a sense of relief. In addition, changes in the size of the sound should be able to reflect changes in body position, the position of the object from far to near, the sound should be small to large, and vice versa.For example, when the self-propelled howitzers virtual maintenance training system, rotating self-propelled howitzers recoil cover extrusion machine copper ring seal liquid should be hit by the virtual sound level to show the cover is securely in place.

\section{Tactile immersion upgrade}

Tactile information is mixed with two kinds of information: one information surface, mainly refers to a variety of information through skin contact with the object being felt; the other is the perception information through muscle movement, refers to the information about the force through the location of the limbs and movement. In general, the surface texture, the soft and hard, the surface roughness can be provided by the surface information produced by the skin , and shape of the object, characterized in elasticity can be provided through the movement of the hand and arm resulting 
perceptual information. As such, in equipment virtual maintenance training system production, while using the hand or other body parts operate the virtual parts, the information should be used between the surface and the body and virtual components generated virtual components to enhance the tactile immersion.

\section{Method of enhancing the behavior system immersion}

\section{To enhance the orientation system immersion}

Orientation system can be used to determine and change the direction of the head body. A comprehensive orientation system,is essential for equipment virtual maintenance training system. Because the equipment virtual maintenance training systems are same as realistic three-dimensional scene, having a clear sense of direction and a sense of place. When participants operate, necessarily requires a real-time system yet complete direction. So that when the freedom to watch, it may selflessly immersed in a virtual environment.

The head tracker is the hardware base of orientation system. Head tracker links system users' movement felling nerve with its visual system. And expand the capabilities of human binocular disparity. It consists of an electromagnetic wave transmitter, receiver, and a serial communication system.Transmitter and stereo helmet solid joint, follow sports helmets. When working, the head movement makes the signal between the transmitter and receiver to produce relative pose changes. Subsequently, changes in the signal transmit into control box, the conversion calculations and generates pose data via the serial communication interface to control the computer. Control computer obtains the operators' head state, thereby generates a corresponding update instruction for the visual system, timely change the local image of the virtual environment. Thus, when the operator in all directions observed, will be able to see the virtual environment in the corresponding direction, and produce real sense of immersion.

\section{Language system immersion upgrade}

Language is mankind's most natural form of communication, if the operator can freely use the language and the environment structured by the virtual reality system to communicate, it will definitely raise the sense of immersion. Microphone is the hardware base of language system, the key technology is the voice recognition technology and voice synthesis technology. By recognizing the participants' language,the user can issue commands to the virtual reality system, or chat with others in a virtual environment.

\section{Expression system immersion upgrade}

Interpersonal communication approach is varied. In addition to language, there are gestures, body potential, expression and so on. In virtual reality, expression system is the use of expression gestures, facial expressions, posture, etc. to convey certain information. Input devices of gesture recognition system is based on the identification data is divided into gloves and based on visual (image) of two sign language recognition system. Based on the data glove gesture recognition system, is the use of data gloves and position tracking device to track and capture the gesture spatial movement of the timing information, to detect more complex hand movements, including the hand position, orientation and curvature of the finger and so on, and gesture can be analyzed based on these information. Therefore it is more practical. Gesture recognition based on visual obtain the visual signals from the visual channel, some systems require staff to wear special color gloves or require a variety of colors to wear gloves to identify various parts of manpower, the general process is: first start using a camera continuously shooting after the motion picture department, and then take each finger contour recognition approach shot on the turn with a boundary feature recognition method to distinguish a small, continuous gestures.

\section{Conclusion}

This paper presents missing immersion in some existing equipment virtual maintenance training system,pointing out the impact of missing immersion on teaching and training effect, introducing the 
meaning and role of immersion, highlighting the visual immersion, auditory immersion,tactile immersion,direction immersion,expression immersion and language immersion six aspects. In short, in teaching and training equipment, we should fully develop the virtual maintenance training system of strong sense of immersion. So in the learning process, it can make a multi-sensory experience, triggering the students a strong sense of immersion in the virtual maintenance training system, gaining a deeper understanding of the learning content.

\section{References}

[1] Li Qin, immersed spread morphology studies, modern communication, 2013 (2)

[2] Wang Yongbo, design elements of virtual scene production , the young writer 2013.1.

[3] Chang Runfang, Zhang Aihua, immersion Promotion Strategy, everyone, 2011 (4)

[4] Tian Xiao,the immersion of virtual culture and visual software, flowers, 2010.1. 